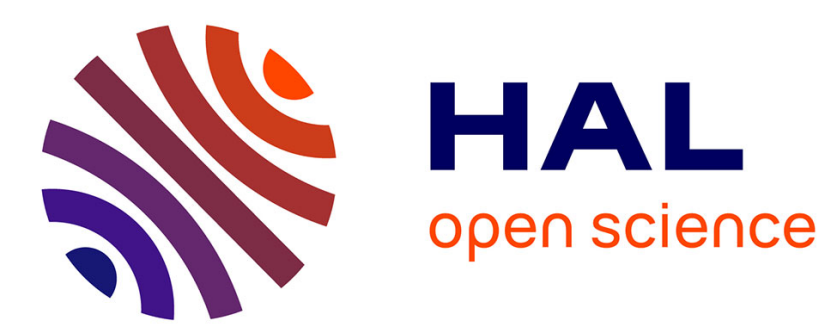

\title{
Turbulent generation of poloidal asymmetries of the electric potential in a tokamak
}

Peter Peter Donnel, X. Garbet, Y. Sarazin, Y. Yuuichi Asahi, F Wilczynski, E Caschera, Guilhem Dif-Pradalier, P Ghendrih, C. Gillot

\section{- To cite this version:}

Peter Peter Donnel, X. Garbet, Y. Sarazin, Y. Yuuichi Asahi, F Wilczynski, et al.. Turbulent generation of poloidal asymmetries of the electric potential in a tokamak. Plasma Physics and Controlled Fusion, In press. hal-01826705

\section{HAL Id: hal-01826705 https://hal.science/hal-01826705}

Submitted on 29 Jun 2018

HAL is a multi-disciplinary open access archive for the deposit and dissemination of scientific research documents, whether they are published or not. The documents may come from teaching and research institutions in France or abroad, or from public or private research centers.
L'archive ouverte pluridisciplinaire HAL, est destinée au dépôt et à la diffusion de documents scientifiques de niveau recherche, publiés ou non, émanant des établissements d'enseignement et de recherche français ou étrangers, des laboratoires publics ou privés. 


\title{
Turbulent generation of poloidal asymmetries of the electric potential in a tokamak
}

\author{
P. Donnel ${ }^{1}$, X. Garbet ${ }^{1}$, Y. Sarazin ${ }^{1}$, Y. Asahi ${ }^{2}$, F.Wilczynski ${ }^{3}$, E. \\ Caschera $^{1}$, \\ G. Dif-Pradalier ${ }^{1}$, P. Ghendrih ${ }^{1}$, C. Gillot ${ }^{4}$ \\ ${ }^{1}$ CEA, IRFM, Saint-Paul-lez-Durance, F-13108, France \\ ${ }^{2}$ Japan Atomic Energy Agency, 178-4-4 Wakashiba, Kashiwa, Chiba, Japan
}

${ }^{3}$ Department of Applied Mathematics, University of Leeds, Leeds LS2 9JT, United Kingdom
${ }^{4}$ Ecole des Ponts ParisTech (hosted by CEA/IRFM), Saint-Paul-lez-Durance, F-13108, France

$29 / 06 / 2018$

\begin{abstract}
Poloidal asymmetries of the $E \times B$ plasma flow are known to play a role in neoclassical transport. One obvious reason is that an asymmetrical potential can produce a flux. Also the associated distribution function may correlate with the magnetic drift velocity to enhance the neoclassical flux. Finally, poloidal variation of the electric potential can produce poloidal asymmetries of the density of an impurity, which in turn may enhance its neoclassical flux. According to conventional neoclassical theory, the level of poloidal asymmetry of the electric potential is expected to be very small. Conversely, poloidal flow asymmetries can be driven by small scale turbulence via non linear coupling, and therefore change this result. In the present work, a general framework for the generation of axisymmetric structures of potential by turbulence is presented. Zonal flows, geodesic acoustic modes and convective cells are described by a single model. This is done by solving the gyrokinetic equation coupled to the quasineutrality equation. This calculation provides a predictive calculation of the frequency spectrum of flows. It also shows that the dominant mechanism comes from zonal flow compression at intermediate frequencies, while ballooning of the turbulence Reynolds stress appears to be the main drive at low frequency.
\end{abstract}

\section{Introduction}

Poloidal asymmetries of the $E \times B$ plasma flow are usually neglected in neoclassical transport. Indeed the predicted level of asymmetry is quite small in conventional neoclassical theory, so that its effect is considered as negligible $[14,12]$. Toroidal momentum transport is however an exception, since it appears to be sensitive to poloidal asymmetries, even at the neoclassical level [23]. This picture changes somewhat if poloidal variations of the electric potential are generated by turbulence. Steady or slowly varying structures may then boost neoclassical fluxes. We will call these structures "poloidal convective cells", since once isolated from axisymmetric flows (mean and zonal flows), and turbulence vortices (helical patterns), poloidal asymmetries of the electric potential are usually characterized by closed streamlines with typically a few lobes in the 
poloidal plane of a tokamak, and no variation in the toroidal direction. In other words, convective cells are modes with a toroidal wave number $n=0$ and poloidal wave numbers $m \neq 0$ (usually $m=1$ prevails). In this classification, zonal flows are $n=0, m=0$ modes, while turbulent vortices are characterized by $n \neq 0$, $m \neq 0$.

One obvious mechanism for the effect of convective cells on neoclassical transport is the modification of the distribution function by the asymmetric potential, that correlates with its associated $E \times B$ electric drift velocity, and/or with the magnetic drift velocity to produce additional fluxes to the standard neoclassical value. Also poloidal variations of the electric potential induce asymmetries of the density of an impurity, which in turn enhance its neoclassical flux [1]. This effect is expected to be particularly large for heavy impurities in core plasmas, since a Boltzmann type response is proportional to the charge number. It was proposed as an explanation for synergies between turbulent and neoclassical fluxes of impurities observed with the GYSELA code $[19,8]$. Another reason for investigating poloidal flow asymmetries is their potential role in turbulence self-organisation, via vortex shearing. The understanding of turbulence self-organization has been an active area of research in the past decades. It is well known that zonal flows are instrumental in these processes. Indeed they are generated by turbulence via the Reynolds stress, and in turn self-regulate turbulence by transferring energy from large scale to small scales [5]. The intensity of Zonal Flows is quite large compared with other helical turbulent structures, because they are not Landau damped [18, 19]. Another player in turbulence self-regulation is the Geodesic Acoustic Mode (GAM) [22] , which is the sum of two axisymmetric components of the potential: a zonal plus an up-down asymmetric component, i.e. a combination of $n=0, m=0$ and $n=0, m= \pm 1$ modes. The whole GAM structure oscillates at a frequency that scales with an acoustic frequency, and is driven by perpendicular flow compression, proportional to the geodesic curvature (hence the name $[22,11]$. These modes are also driven by turbulence via mode coupling, and can back-react on the turbulence background. GAMs have been shown however to be less effective than zonal flows in this retro-action process, due to their high frequency [10]. Moreover they are Landau damped in contrast with Zonal Flows. One open issue therefore is whether low frequency poloidal flow asymmetries can have an impact on turbulence in a way similar to Zonal Flows, as proposed in [20]. Let us note that some recent turbulence simulations seem to indicate that this effect is weak, while the synergy with neoclassical fluxes appears to be prominent in most transport channels, except momentum transport [2].

Quite curiously a predictive calculation of the dynamics and amplitude of poloidal convective cells is not available in the literature, to the authors knowledge. We note a previous attempt [9], originally intended to explain the anti-correlation between fluxes of momentum driven by $E \times B$ and magnetic drifts seen in several gyrokinetic simulations. However this calculation does not account for the coexistence of convective cells with zonal flows and GAMs, an important expected character of a comprehensive theory. We propose here a model that allows a simultaneous estimate of Zonal Flows, GAMs and convective cells. A complete theory of convective cells generation is out of scope here, as it requires to keep the whole dynamics of trapped and passing particles and the inclusion of collisions, to recover the correct dynamics of zonal flows at low frequency $[18,13]$. Instead we use here a simpler model that keeps only passing particles, and neglects the effect of trapped particles. The Vlasov gyrokinetic equation can then be solved for $n=0, m=0, \pm 1$ modes, assuming non linear terms are given. The latter corresponds to the turbulence drive, and can be shown to be related to the turbulent Reynolds stress. This method allows one to recover exactly the GAMs dispersion relation, and to compute poloidal convective cells in an intermediate range of frequencies that goes down to the trapping frequency. Two mechanisms of generation are identified: the generation of cells due to compressional effect of zonal flows, and the poloidal asymmetry of the Reynolds stress due to turbulence ballooning. The first one is dominant in the range of frequency of the order of the curvature frequency, while the latter appears to be prominent at very low frequency. One interesting finding is that the asymmetry of poloidal convective cells is in-out at low frequencies, and up-down at intermediate and high frequencies (GAMs).

The remainder of the paper is organised as follows. The method used to calculated poloidal asymmetries of the electric potential is described in section 2. The link between the turbulence drive term and the turbulent Reynolds stress is established in section 3. The main properties of poloidal convective cells are given in section 4 , and a conclusion follows in section 5 . 


\section{Poloidal asymmetries of the electric potential}

\subsection{Equilibrium field and particle trajectories}

The unperturbed part of the magnetic field is written in Clebsch representation,

$$
\mathbf{B}_{e q}=\nabla \alpha \times \nabla \psi
$$

where $\alpha=\varphi-q(\psi) \theta$, and $q(\psi)$ is the safety factor. In the special case of a large aspect ratio tokamak with circular concentric magnetic surfaces labeled by their minor radius $r$, the function $\psi$ is given by $\frac{d \psi}{d r}=\frac{r}{q(r)} \frac{B_{0}}{R_{0}}$, where $R_{0}$ is the major radius. The inverse aspect ratio $\epsilon=\frac{r}{R_{0}}$ is considered as a small parameter, and calculations are done at first order in $\epsilon$. The unperturbed equation of motion is integrable, since there exists three invariants of motion, namely the unperturbed Hamiltonian $H_{e q}$, the magnetic moment $\mu$, and the kinetic toroidal momentum $P_{\varphi}=-e \psi+m R v_{\varphi}$ where $v_{\varphi}=\frac{B_{\varphi}}{B} v_{\|}$. This implies that a set of conjugated Hamiltonian variables can be built. The first couple is the cyclotron pulsation and the corresponding action is $\frac{m}{e} \mu$. It is of little use since in gyrokinetics the Hamiltonian does not depend on the cyclotron phase, thus leaving $\mu$ invariant. In the spirit of the ballooning representation, we choose the second and third variables as $\theta$ and $\alpha$, where $\theta$ plays the role of a coordinate along the unperturbed field lines. The corresponding actions are the parallel adiabatic action $J_{\|}=\frac{1}{2 \pi} \int \frac{B d \theta}{\mathrm{B} \cdot \nabla \theta} m v_{\|}$, and the canonical toroidal momentum $P_{\varphi}$. The second Hamiltonian frequency is a poloidal transit frequency $\Omega_{t}=\frac{v_{\|}}{q(r) R_{0}}$, while the third Hamiltonian frequency is related to curvature and will be neglected here as it plays little role for passing particles. The action $J_{\|}$is not handy, and can be efficiently replaced by the unperturbed Hamiltonian $H_{e q} . \Omega_{t}$ is a poloidal transit frequency in quasi-cylindrical geometry.

\subsection{Evolution equation for the axisymmetric mode}

function is written as $\bar{F}=F_{e q}+\bar{f}$, where the unperturbed distribution function $F_{e q}$ depends on the motion invariants only $\left(H_{e q}, P_{\varphi}, \mu, \operatorname{sign}\left(v_{\|}\right)\right)$, while $\bar{f}$ also depends on $\theta$ and $\alpha$. Moreover the perturbed distribution function is split in adiabatic plus resonant parts $\bar{f}=-\frac{\bar{h}}{T_{e q}} F_{e q}+\bar{g}$, where the "temperature" $\frac{1}{T_{e q}}=-\frac{\partial}{\partial H_{e q}} \ln F_{e q}$ depends on the motion invariants only. $T_{e q}$ is the usual local temperature when $F_{e q}$ is a Maxwellian. This is the case that will be considered in the following. The resonant part is solution of the equation

$$
\begin{aligned}
\frac{\partial \bar{g}}{\partial t}-\{\bar{H}, \bar{g}\} & =\frac{\partial \bar{g}}{\partial t}+v_{\|} \nabla_{\|} \bar{g}+\overline{\mathbf{v}}_{E} \cdot \nabla \bar{g}+\overline{\mathbf{v}}_{D} \cdot \nabla \bar{g} \\
& =\frac{F_{e q}}{T_{e q}}\left(\frac{\partial}{\partial t}+\Omega^{*} \frac{\partial}{\partial \alpha}\right) \bar{h}
\end{aligned}
$$

where $\Omega^{*}=T_{e q} \frac{\partial \ln F_{M}}{\partial P_{\varphi}}$ is the kinetic diamagnetic frequency and the relevant $\bar{h} / T_{e q} \ll 1$ limit has been considered. We now separate the electric potential between an axisymmetric component $\langle\phi\rangle_{\varphi}(\psi, \theta)$ and a helical perturbation $\tilde{\phi}(\psi, \theta, \varphi)$, i.e. $\phi=\langle\phi\rangle_{\varphi}+\tilde{\phi}$. The axisymmetric part is written as a Fourier series

$$
\langle\phi\rangle_{\varphi}(\psi, \theta)=\sum_{M \Omega} \phi_{M \Omega}(\psi) \exp \{i(M \theta-\Omega t)\}
$$

We use a ballooning representation for the non axisymmetric part $[21,17$, 4], i.e.

$$
\begin{aligned}
\tilde{\phi}(\psi, \theta, \varphi, t) & =\sum_{\mathbf{k} \omega} \phi_{n \omega}\left(\theta_{k}, \theta+2 p \pi\right) \\
& \times \exp \left\{i n\left[\varphi-q(\psi)\left(\theta-\theta_{k}+2 p \pi\right)\right]-i \omega t\right\}
\end{aligned}
$$

where $\mathbf{k}=\left(n, \theta_{k}, p\right)$. The integer $p$ spans the interval $]-\infty,+\infty[$, however for a ballooned turbulence, it is sufficient to keep the component $p=0$ only. In this case, a useful reformulation of Eq.(2) in the set of field aligned coordinates is

$$
\tilde{\phi}(\psi, \alpha, \theta, t)=\sum_{\mathbf{k} \omega} \phi_{\mathbf{k} \omega}(\theta) \exp \left\{i\left[n \theta_{k} q(\psi)+n \alpha-\omega t\right]\right\}
$$


Note the different notations for the frequencies $\Omega$ and poloidal wave numbers $M$ of the axisymmetric potential on the one hand, and the toroidal wave numbers and frequencies $n, \omega$ of the helical perturbations. We omit to specify $n=0$ for the axisymmetric perturbation, which is made distinct from helical fluctuations by the different notations. Taking the Fourier transform in radial position and assuming $\frac{M}{r} \ll K_{r}$, one can compute the linear solution of the Vlasov equation for the axisymmetric perturbations

$$
\begin{aligned}
& -i\left(\Omega-M \Omega_{t}\right) \bar{g}_{M \Omega}+\Omega_{D}\left(\bar{g}_{M+1, \Omega}-\bar{g}_{M-1, \Omega}\right) \\
& =-i \Omega \frac{F_{e q}}{T_{e q}} \bar{h}_{M \Omega}-\left[\tilde{\bar{v}}_{E} \cdot \nabla \tilde{\bar{g}}\right]_{M \Omega}
\end{aligned}
$$

where $\Omega_{D}=\frac{K_{r} \rho_{i} v_{T}}{4 R_{0}}\left(2 \frac{v_{\|}^{2}}{v_{T}^{2}}+\frac{v_{\perp}^{2}}{v_{T}^{2}}\right)$. Note that we have chosen the direct basis to be $\left(e_{r}, e_{\theta}, e_{\varphi}\right)$. The non linear term $\left[\left\langle\overline{\mathbf{v}}_{E}\right\rangle_{\varphi} \cdot \nabla\langle\bar{g}\rangle_{\varphi}\right]_{M \Omega} \simeq 0$ is neglected, since 1st order terms only are kept. However this term may play a role in the dynamics, and could actually provide a saturation mechanism that does not have a counter-part for zonal flows. Keeping only the modes $M=-1,0,1$, the relation (3) can be written

$$
\{\bar{g}\}=-i \Omega G^{-1} \frac{F_{e q}}{T_{e q}}\{\bar{h}\}-G^{-1}\left\{\left[\tilde{\bar{v}}_{E} \cdot \nabla \tilde{\bar{g}}\right]\right\}
$$

where

$$
\begin{aligned}
\{\bar{g}\} & =\left(\begin{array}{c}
\bar{g}_{-1, \Omega} \\
\bar{g}_{0, \Omega} \\
\bar{g}_{1, \Omega}
\end{array}\right) \\
\{\bar{h}\} & =\left(\begin{array}{c}
\bar{h}_{-1, \Omega} \\
\bar{h}_{0, \Omega} \\
\bar{h}_{1, \Omega}
\end{array}\right) \\
\left\{\left[\tilde{\bar{v}}_{E} \cdot \nabla \tilde{\bar{g}}\right]\right\} & =\left(\begin{array}{c}
{\left[\tilde{\bar{v}}_{E} \cdot \nabla \tilde{\bar{g}}\right]_{-1, \Omega}} \\
{\left[\tilde{\bar{v}}_{E} \cdot \nabla \tilde{\bar{g}}\right]_{0, \Omega}} \\
{\left[\tilde{\bar{v}}_{E} \cdot \nabla \overline{\bar{g}}\right]_{1, \Omega}}
\end{array}\right)
\end{aligned}
$$

and

$$
G=\left(\begin{array}{ccc}
-i \Omega_{+} & \Omega_{D} & 0 \\
-\Omega_{D} & -i \Omega & \Omega_{D} \\
0 & -\Omega_{D} & i \Omega_{-}
\end{array}\right)
$$

where $\Omega_{ \pm}=\Omega_{t} \pm \Omega$. The determinant of the $G$ matrix is $\operatorname{det} G=-i \Omega\left(\Omega_{+} \Omega_{-}+2 \Omega_{D}^{2}\right)$. A useful quantity is the transpose of its comatrix, given by

$$
\operatorname{com} G^{t}=\left(\begin{array}{ccc}
\Omega_{-} \Omega_{+} \Omega_{D}^{2} & -i \Omega_{D} \Omega_{-} & \Omega_{D}^{2} \\
i \Omega_{D} \Omega_{-} & \Omega_{+} \Omega_{-} & i \Omega_{D} \Omega_{+} \\
\Omega_{D}^{2} & -i \Omega_{D} \Omega_{+} & -\Omega_{+} \Omega_{+} \Omega_{D}^{2}
\end{array}\right)
$$

Assuming adiabatic electrons and keeping a single hydrogenoid (charge number $Z=1$ ) ion species, the quasi-neutrality equation reads

$$
\frac{N_{e q}}{T_{e q}}[1+(1-\delta(M)) \tau]\{h\}=\int \mathcal{J}\{\bar{g}\} d^{3} v
$$

where $\tau=\frac{T_{i}}{T_{e}}$ is the ratio of ion to electron temperatures, and $\mathcal{J}$ is the gyro-average operator. Combining the quasi-neutrality condition Eq.(5) with the equation on $\{\bar{g}\}$ Eq.(4), and assuming the gyroaverage operator commutes with all quantities at all frequencies, one gets

$$
\begin{aligned}
& \frac{N_{e q}}{T_{e q}}[1+(1-\delta(M)) \tau]\{h\}+i \Omega \int G^{-1} \frac{F_{e q}}{T_{e q}} \mathcal{J}^{2}\{h\} d^{3} v \\
& =-\int G^{-1} \mathcal{J}\left\{\left[\tilde{\bar{v}}_{E} \cdot \nabla \tilde{\bar{g}}\right]\right\} d^{3} v
\end{aligned}
$$


Eq.(6) is the formal solution that gives the amplitude of the poloidal convective cells. It can be expressed as $\mathcal{E} \cdot\{h\}=\{S\}$. Defining $\langle\cdot\rangle_{v}=\int \cdot \frac{F_{e q}}{N_{e q}} d^{3} v$ and using the fact that $\langle g\rangle_{v}=0$ whatever $g$ odd with respect to $v_{\|}$, one can show that

$$
\mathcal{E}=\frac{N_{e q}}{T_{e q}}\left(\begin{array}{ccc}
\mathcal{E}_{a} & -i \mathcal{E}_{c} & \mathcal{E}_{d} \\
i \mathcal{E}_{c} & \mathcal{E}_{b} & -i \mathcal{E}_{c} \\
\mathcal{E}_{d} & i \mathcal{E}_{c} & \mathcal{E}_{a}
\end{array}\right)
$$

where

$$
\begin{aligned}
& \mathcal{E}_{a}=1+\tau+L_{0}(\Omega)-L_{2}(\Omega) \\
& \mathcal{E}_{b}=\left\langle 1-\mathcal{J}^{2}\right\rangle_{v}+2 L_{2}(\Omega) \\
& \mathcal{E}_{c}=L_{1}(\Omega) \\
& \mathcal{E}_{d}=-L_{2}(\Omega)
\end{aligned}
$$

where

$$
L_{j}(\Omega)=\left\langle\frac{\Omega^{2-j} \Omega_{D}^{j}}{\Omega_{+} \Omega_{-}+2 \Omega_{D}^{2}} \mathcal{J}^{2}\right\rangle_{v}
$$

Assuming that $\mathcal{J}\left[\tilde{\bar{v}}_{E} \cdot \nabla \tilde{\bar{g}}\right]_{M, \Omega}$ is an even function of $v_{\|}$, it can be shown that

$$
\{S\}=\frac{1}{\Omega}\left(\begin{array}{c}
S_{1,0}+i\left(S_{0,-1}-S_{2,-1}-S_{2,1}\right) \\
-i S^{\prime}-S_{1,-1}+S_{1,1}+2 i S_{2,0} \\
-S_{1,0}+i\left(S_{0,1}-S_{2,-1}-S_{2,1}\right)
\end{array}\right)
$$

where

$$
S_{j, M}=\int \frac{\Omega^{2-j} \Omega_{D}^{j}}{\left(\Omega_{+} \Omega_{-}+2 \Omega_{D}^{2}\right)} \mathcal{J}\left[\tilde{\bar{v}}_{E} \cdot \nabla \tilde{\bar{g}}\right]_{M, \Omega} d^{3} v
$$

and

$$
S^{\prime}=\int \mathcal{J}\left[\tilde{\bar{v}}_{E} \cdot \nabla \tilde{\bar{g}}\right]_{0, \Omega} d^{3} v
$$

We introduce typical frequencies $\Omega_{t h}=\frac{v_{T}}{q(r) R_{0}}, \Omega_{D t h}=\frac{K_{r} \rho_{i} v_{T}}{R_{0}}=q K_{r} \rho_{i} \Omega_{t h}$, and two dimensionless parameters $\tilde{K}=K_{r} \rho_{i}$ and $\tilde{\Omega}=\frac{\Omega}{\Omega_{t h}}$. In the following we assume $\tilde{K} \ll 1$. In this limit, one has $\left\langle 1-\mathcal{J}^{2}\right\rangle_{v}=$ $\tilde{K}^{2}$.

\subsubsection{GAMs}

We assume that $\tilde{\Omega}=O(1)$. In this case, noting that $\mathcal{J}=1+O\left(\tilde{K}^{2}\right)$, and the fact that whatever the function $f$ even with respect to $v_{\|}$

$$
\left\langle\frac{f}{\Omega_{+} \Omega-}\right\rangle_{v}=\frac{1}{2 \Omega}\left(\left\langle\frac{f}{\Omega_{-}}\right\rangle_{v}-\left\langle\frac{f}{\Omega_{+}}\right\rangle_{v}\right)=\frac{1}{\Omega}\left\langle\frac{f}{\Omega-}\right\rangle_{v}
$$

one can show that the coefficients $L_{j}(\Omega)(8)$ become at leading order

$$
\begin{aligned}
& L_{0}(\Omega)=\frac{\tilde{\Omega}}{\sqrt{2}} \zeta\left(\frac{\tilde{\Omega}}{\sqrt{2}}\right) \\
& L_{1}(\Omega)=\frac{q \tilde{K}}{2 \sqrt{2}}\left[\sqrt{2} \tilde{\Omega}+\left(1+\tilde{\Omega}^{2}\right) \zeta\left(\frac{\tilde{\Omega}}{\sqrt{2}}\right)\right] \\
& L_{2}(\Omega)=\frac{q^{2} \tilde{K}^{2}}{4}\left[3+\tilde{\Omega}^{2}+\frac{\sqrt{2}}{\tilde{\Omega}}\left(\frac{\tilde{\Omega}^{4}}{2}+\tilde{\Omega}^{2}+1\right) \zeta\left(\frac{\tilde{\Omega}}{\sqrt{2}}\right)\right]
\end{aligned}
$$

where $\zeta(z)=\frac{1}{\sqrt{\pi}} \int_{-\infty}^{\infty} \frac{e^{-x^{2}}}{x-z} d x$ is the plasma dispersion function. The simplification (9) implies also $S_{j, M}=\int \frac{\Omega^{1-j} \Omega_{D}^{j}}{\Omega_{-}} \mathcal{J}\left[\tilde{\bar{v}}_{E} \cdot \nabla \tilde{\bar{g}}\right]_{M, \Omega} d^{3} v$ at leading order. We define the following quantities 


$$
\begin{aligned}
X_{0} & =\frac{N_{e q}}{T_{e q}}\left[1+\tau+L_{0}(\Omega)\right] \\
X_{1} & =\frac{N_{e q}}{T_{e q}} L_{1}(\Omega) \\
X_{2} & =\frac{N_{e q}}{T_{e q}}\left[\tilde{K}^{2}+2 L_{2}(\Omega)\right]
\end{aligned}
$$

where the functions $L_{j}(\Omega)$ are given by Eq.(10) when $\tilde{\Omega}=O(1)$. At this stage, one should remark that notations have been chosen such that $X_{j}=O\left(\tilde{K}^{j}\right)$ and $S_{j, M}=O\left(\tilde{K}^{j}\right)$. These properties greatly simplify the computation. With these notations, the determinant of $\mathcal{E}$ is given by

$$
\operatorname{det} \mathcal{E}=X_{0}\left[X_{0} X_{2}-2 X_{1}^{2}\right]+O\left(\tilde{K}^{4}\right)
$$

One should notice that the relation $\operatorname{det} \mathcal{E}=0$ gives the kinetic GAM dispersion relation. Indeed, replacing the coefficients $X_{i}(11)$ in the expression Eq.((12)) leads to

$$
\begin{aligned}
& \zeta\left(\frac{\tilde{\Omega}}{\sqrt{2}}\right)\left\{\frac{\tilde{\Omega}}{\sqrt{2}}+\frac{q^{2}}{\sqrt{2}}\left[\frac{\tau}{2} \tilde{\Omega}^{3}+\left(\frac{3}{2}+\tau\right) \tilde{\Omega}+\frac{1+\tau}{\tilde{\Omega}}\right]\right\} \\
& +1+\tau+\frac{\tau}{2} q^{2} \tilde{\Omega}^{2}+\frac{3}{2} q^{2}(1+\tau)+\frac{q^{2}}{4} \zeta^{2}\left(\frac{\tilde{\Omega}}{\sqrt{2}}\right)=0
\end{aligned}
$$

which is the fully kinetic dispersion relation of $\operatorname{GAMs}[24,19,25]$. Assuming that the order of $\mathcal{J}\left[\tilde{\bar{v}}_{E} \cdot \nabla \tilde{\bar{g}}\right]_{M, \Omega}$ is independent of $M$, it appears that

$$
\begin{aligned}
i \Omega h_{-1, \Omega} \operatorname{det} \mathcal{E} & =i X_{0} X_{1} S^{\prime}+X_{0} X_{1}\left(S_{1,-1}-S_{1,1}\right) \\
& +\left(X_{1}^{2}-X_{0} X_{2}\right) S_{0,-1}+X_{1}^{2} S_{0,1}+O\left(\tilde{K}^{3}\right) \\
i \Omega h_{1, \Omega} \operatorname{det} \mathcal{E} & =-i X_{0} X_{1} S^{\prime}+X_{0} X_{1}\left(S_{1,1}-S_{1,-1}\right) \\
& +\left(X_{1}^{2}-X_{0} X_{2}\right) S_{0,-1}+X_{1}^{2} S_{0,1}+O\left(\tilde{K}^{3}\right) \\
i \Omega h_{0, \Omega} \operatorname{det} \mathcal{E} & =X_{0}^{2} S^{\prime}+i X_{0}^{2}\left(S_{1,1}-S_{1,-1}\right)+O\left(\tilde{K}^{2}\right)
\end{aligned}
$$

At leading order, one has $h_{-1, \Omega}=-h_{1, \Omega}$. Hence the mode poloidal dependence is $\sin \theta$ (up-down asymmetry), as expected for GAMs. We will show in the section 3 that $S^{\prime}$ is linked to the turbulent Reynolds stress tensor. This calculation shows that for the GAM limit, the side bands $M= \pm 1$ are mainly fed via the toroidal coupling with the mode $M=0$. This result is new and non trivial, as one could have also expected a direct feeding via a source of the form $\mathcal{J}\left[\tilde{\bar{v}}_{E} \cdot \nabla \tilde{\bar{g}}\right]_{1, \Omega}$ to be competitive.

\subsubsection{Pololoidal convective cells}

consider the low frequency limit the limit $\tilde{\Omega} \ll \tilde{K} \ll 1$. We restrict the analysis to the first order terms in $\tilde{\Omega}$. Using the property

$$
\left\langle\frac{f}{\Omega_{t}^{2}+2 \Omega_{D}^{2}}\right\rangle_{v}=-\frac{i}{\sqrt{2}}\left\langle\frac{f}{\Omega_{D}\left(\Omega_{t}-i \sqrt{2} \Omega_{D}\right)}\right\rangle_{v}
$$

for any even function $f\left(v_{\|}\right)$, it turns out that at leading order the functions $L_{j}$ (8) become

$$
\begin{aligned}
& L_{0}(\Omega)=-\sqrt{\pi} \frac{\tilde{\Omega}^{2}}{q \tilde{K}} \ln \left(i \frac{\tilde{\Omega}}{q \tilde{K}}\right) \\
& L_{1}(\Omega)=\frac{\sqrt{\pi}}{2} \tilde{\Omega} \\
& L_{2}(\Omega)=\frac{\sqrt{\pi}}{4} q \tilde{K}
\end{aligned}
$$


The simplification (13) leads to the redefinition of the source term $S_{j, M}=-\frac{i}{\sqrt{2}} \int \frac{\Omega^{2-j} \Omega_{D}^{j-1}}{\Omega_{t}-i \sqrt{2} \Omega_{D}} \mathcal{J}\left[\tilde{\bar{v}}_{E} \cdot \nabla \tilde{\bar{g}}\right]_{M, \Omega} d^{3} v=$ $O\left(\tilde{K}^{j-1}\right) \times O\left(\tilde{\Omega}^{2-j}\right)$. Using this ordering, one can compute the potential at leading order

$$
\begin{aligned}
i \Omega h_{-1, \Omega} & =\frac{T_{e q}}{N_{e q}} \frac{1}{(1+\tau) q \tilde{K}}\left(i \tilde{\Omega} S^{\prime}+q \tilde{K}\left(S_{2,-1}+S_{2,1}\right)\right) \\
i \Omega h_{1, \Omega} & =\frac{T_{e q}}{N_{e q}} \frac{1}{(1+\tau) q \tilde{K}}\left(-i \tilde{\Omega} S^{\prime}+q \tilde{K}\left(S_{2,-1}+S_{2,1}\right)\right) \\
i \Omega h_{0, \Omega} & =\frac{T_{e q}}{N_{e q}} \frac{2 S^{\prime}}{\sqrt{\pi} q \tilde{K}}
\end{aligned}
$$

Two limit cases are found. In the intermediate frequency regime $\tilde{K}^{2} \ll \tilde{\Omega} \ll \tilde{K}$, one gets

$$
-h_{-1, \Omega}=h_{1, \Omega}=-\frac{T_{e q}}{N_{e q}} \frac{S^{\prime}}{(1+\tau) q \tilde{K} \Omega_{t h}}
$$

This corresponds to a $\sin (\theta)$ poloidal structure (up-down asymmetry). It appears that the main drive comes from the source $S^{\prime}$ that is a $N=0, M=0$ component of the source. It is shown in the next section that it is related to the flux surface average of the turbulent Reynolds stress, i.e. the same source as zonal flows. The underlying mechanism is flow compressibility, that results in a pumping of $N=0, M= \pm 1$ modes by $N=0, M=0$ zonal flows - i.e. a sideband effect.

The second limit corresponds to quasi-stationary structures, i.e. very low frequencies $\tilde{\Omega} \ll \tilde{K}^{2}$, for which at leading order

$$
h_{-1, \Omega}=h_{1, \Omega}=\frac{T_{e q}}{N_{e q}} \frac{\left(S_{2,-1}+S_{2,1}\right)}{i \Omega(1+\tau)}
$$

It corresponds to a $\cos (\theta)$ poloidal variation (in-out asymmetry). The source terms $S_{2, \pm 1}$ corresponds to $M= \pm 1$ components of the turbulent source. Hence the drive rather comes from turbulence ballooning, which induces in turn a ballooning of the turbulence source, i.e. non zero poloidal harmonics of the turbulent drive.

\section{Link between source terms and turbulent Reynolds stress tensor}

is often proportional to the source term $S^{\prime}$. In this part we establish the connection between this quantity and the Reynolds stress tensor. In other words we demonstrate a kinetic version of the Taylor identity [16]. The first step, detailed in Appendix A consists in demonstrating that the turbulent source $\mathcal{J}\left[\overline{\mathbf{v}}_{E} \cdot \nabla \bar{g}\right]_{M \Omega}$ can be written as a flux, $\mathcal{J}\left[\overline{\mathbf{v}}_{E} \cdot \nabla \bar{g}\right]_{M \Omega}=\frac{\partial \Gamma_{\Pi}}{\partial \psi}$, where the flux $\Gamma_{\Pi}$ is a flux of vorticity

$$
\Gamma_{\Pi}=\int_{0}^{T} \frac{d t}{T} \int_{0}^{2 \pi} \frac{d \theta}{2 \pi} e^{-i M \theta+i \Omega t} \int_{0}^{2 \pi} \frac{d \alpha}{2 \pi} \mathcal{J}\left(\overline{\mathcal{E}}_{\alpha} \bar{f}\right)
$$

and $\overline{\mathcal{E}}_{\alpha}=\frac{\partial(\mathcal{J} \phi)}{\partial \alpha}$ is the $\alpha$ component of the gyroaverage electric field. Therefore

$$
S^{\prime}=\frac{\partial}{\partial \psi}\left[\int_{0}^{T} \frac{d t}{T} e^{i \Omega t} \int_{0}^{2 \pi} \frac{d \theta}{2 \pi} \sigma\right]
$$

where $\sigma=\int \frac{d \alpha}{2 \pi} \int d^{3} v \mathcal{J}\left(\overline{\mathcal{E}}_{\alpha} \bar{f}\right)$. One is left with the computation of $\sigma$. From now on, the calculation is restricted to the wavelength limit $k_{\perp} \rho_{i}<1$. Using $\mathcal{J} \simeq 1+\frac{m \mu}{2 e^{2} B_{e q}} \nabla_{\perp}^{2}$, one finds

$$
\mathcal{J}\left(\overline{\mathcal{E}}_{\alpha} \bar{f}\right)=\mathcal{E}_{\alpha} \mathcal{J} \bar{f}+\frac{m \mu}{e^{2} B_{e q}} \bar{f} \nabla_{\perp}^{2} \mathcal{E}_{\alpha}+\frac{m \mu}{e^{2} B_{e q}} \nabla_{\perp} \mathcal{E}_{\alpha} \cdot \nabla_{\perp} \bar{f}
$$

It is convenient to calculate the contribution of $\mathcal{J} \bar{f}$ by using the Poisson equation that writes

$$
\frac{N_{e q} e^{2}}{T_{e, e q}}\left(\phi-\langle\phi\rangle_{\psi}\right)-\nabla \cdot\left(\frac{N_{e q} m}{B_{e q}^{2}} \nabla_{\perp} \phi\right)=e \int \mathrm{d}^{3} \mathbf{v} \mathcal{J} \bar{f}
$$


Then

$$
\begin{aligned}
\int d^{3} v \mathcal{J}\left(\overline{\mathcal{E}}_{\alpha} \bar{f}\right) & =\mathcal{E}_{\alpha} \frac{N_{e q} e}{T_{e, e q}}\left(\phi-\langle\phi\rangle_{\psi}\right) \\
& -\mathcal{E}_{\alpha} \nabla \cdot\left(\frac{N_{e q} m}{e B_{e q}^{2}} \nabla_{\perp} \phi\right) \\
& +\frac{m}{e^{2} B_{e q}^{2}} \nabla \cdot\left(\bar{P}_{\perp} \nabla_{\perp} \mathcal{E}_{\alpha}\right)
\end{aligned}
$$

where $\bar{P}_{\perp}=\int d^{3} v \mu B_{e q} \bar{f}$. The next step consists in integrating with respect to $\alpha$. The terms proportional to $\left(\phi-\langle\phi\rangle_{\psi}\right)$ is obviously not contributing. The terms $\frac{N_{e q} m}{e B_{e q}^{2}}$ are varying at large scales and so their derivatives can be neglected. Then

$$
\sigma=\frac{N_{e q} m}{e B_{e q}^{2}} \int_{0}^{2 \pi} \frac{d \alpha}{2 \pi}\left[\begin{array}{c}
\frac{1}{e N_{e q}} \nabla \cdot\left(\bar{P}_{\perp} \nabla_{\perp} \mathcal{E}_{\alpha}\right) \\
-\mathcal{E}_{\alpha} \nabla \cdot\left(\nabla_{\perp} \phi\right)
\end{array}\right]
$$

Using integration by part and the fact that $\mathcal{E}_{\alpha} \nabla \cdot\left(\nabla_{\perp} \phi\right)=\nabla \cdot\left(\mathcal{E}_{\alpha} \nabla_{\perp} \phi\right)-\frac{1}{2} \partial_{\alpha}\left|\nabla_{\perp} \phi\right|^{2}$. One can show that

$$
\sigma=-\frac{N_{e q} m}{e B_{e q}^{2}} \int_{0}^{2 \pi} \frac{d \alpha}{2 \pi} \nabla \cdot\left[\left(\mathcal{E}_{\alpha}+\frac{\mathcal{P}_{\alpha}}{e N_{e q}}\right) \nabla_{\perp} \phi\right]
$$

where $\mathcal{P}_{\alpha}=\frac{\partial \bar{P}_{\perp}}{\partial \alpha}$. We then use the definition of the divergence in ballooning coordinates

$$
\nabla \cdot \mathbf{U}=\frac{1}{\sqrt{g}} \frac{\partial}{\partial \psi}(\sqrt{g} \mathbf{U} \cdot \nabla \psi)+\frac{1}{\sqrt{g}} \frac{\partial}{\partial \alpha}(\sqrt{g} \mathbf{U} \cdot \nabla \alpha)
$$

where $\sqrt{g}=\frac{1}{\mathbf{B}_{e q} \cdot \nabla \theta}$ is the Jacobian. The $\psi$ dependence of $g$ can be neglected. One then finds

$$
\sigma=-\frac{\partial}{\partial \psi}\left[\frac{N_{e q} m}{e B_{e q}^{2}} \int \frac{d \alpha}{2 \pi} \times\left(\begin{array}{c}
\left(\mathcal{E}_{\alpha}+\frac{\mathcal{P}_{\alpha}}{e N_{e q}}\right) \\
\left.\left.\mathcal{E}_{\psi}|\nabla \psi|^{2}+\mathcal{E}_{\alpha} \nabla \psi \cdot \nabla \alpha\right)\right]
\end{array}\right]\right.
$$

where $\mathcal{E}_{\psi}=\frac{\partial \phi}{\partial \Psi}$. We note that $\nabla \psi \cdot \nabla \alpha=-q \nabla \psi \cdot \nabla \theta-\frac{\partial q}{\partial \psi} \theta|\nabla \psi|^{2}$. Moreover one has the following properties in ballooning representation

$$
\begin{aligned}
\phi & =\sum_{\mathbf{k}} \phi_{\mathbf{k}}(\theta, t) \exp \left\{i\left[n \theta_{k} q(\psi)+n \alpha\right]\right\} \\
\mathcal{E}_{\psi} & =\sum_{\mathbf{k}} i n \frac{\partial q}{\partial \psi} \theta_{k} \phi_{\mathbf{k}}(\theta, t) \exp \left\{i\left[n \theta_{k} q(\psi)+n \alpha\right]\right\} \\
\mathcal{E}_{\alpha} & =\sum_{\mathbf{k}} i n \phi_{\mathbf{k}}(\theta, t) \exp \left\{i\left[n \theta_{k} q(\psi)+n \alpha\right]\right\}
\end{aligned}
$$

where it is reminded that $n \phi_{\mathbf{k}} \gg \frac{\partial \phi_{\mathbf{k}}}{\partial \theta}$. Similar relations can be derived for the perpendicular pressure. Using also the limit $\Omega \lll \omega$, one gets

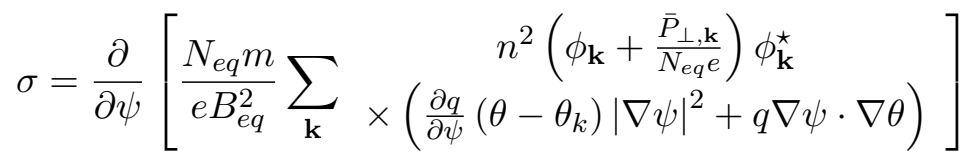

If we moreover assume the distribution function is mainly advected by the turbulent $E \times B$ drift velocity, then $\bar{f}_{\mathbf{k}, \omega}=-\frac{F_{e q}}{T_{e q}} \frac{n \Omega_{\star}}{\omega} h_{\mathbf{k}, \omega}$, and one has

$$
\sigma=\frac{\partial}{\partial \psi}\left[\frac{N_{e q} m}{e B_{e q}^{2}} \sum_{\mathbf{k}} \times\left(\frac{\partial q}{\partial \psi}\left(\theta-\theta_{k}\right)|\nabla \psi|^{2}+q \nabla \psi \cdot \nabla \theta\right)\right]
$$


It then appears that $\sigma$ is close to $-\frac{\partial}{\partial r}\left(\frac{\partial \psi}{\partial r} \frac{N_{e q} m}{e B_{e q}} \Pi_{R S}\right)$, where $\Pi_{R S}=\left\langle v_{E r}\left(v_{E \theta}+V_{\theta}^{*}\right)\right\rangle$ is the turbulent Reynolds stress tensor, with FLR effects. It is a function of radius $r$, poloidal angle $\theta$, and time $t$. Using Eq.(15), source $S^{\prime}$ is then shown to be related to the $M=0$ component of the stress tensor

$$
S^{\prime}=-\frac{N_{e q} e B_{e q}}{T_{e q}} \rho_{i}^{2} \frac{\partial^{2}}{\partial r^{2}} \Pi_{R S, 0}(r, \Omega)
$$

where $\rho_{i}$ is the thermal ion gyroradius. Radial variations of $\frac{\partial \psi}{\partial r}$ and all equilibrium quantities have been ignored compared to variations of the turbulence intensity to get this estimate. Other source components $S_{i, M}$ are more difficult to estimate accurately, but can be shown to scale as the Reynolds stress tensor.

\section{Discussion}

\subsection{Phase and amplitude of poloidal convective cells}

examine the results obtained for poloidal convective cells. In the intermediate frequency regime $\Omega \leq \Omega_{D}$, the level of of the $M=1$ mode is given by

$$
\frac{\phi_{1, \Omega}}{B_{e q}}=-\frac{\phi_{-1, \Omega}}{B_{e q}}=-K_{r} \rho_{i} \frac{\Pi_{R S, 0}(\Omega)}{(1+\tau) \frac{v_{T}}{R_{0}}}
$$

This corresponds to an up-down asymmetric potential, i.e. $\sin \theta$ structure. This level is finite. For $\Omega \sim \Omega_{D}$, the level of zonal flow is given by $\phi_{0, \Omega} \sim \frac{\Pi_{R S}}{v_{T} / R_{0}}$. Hence the level of poloidal convective cell is smaller than a typical saturated zonal flow by a factor $K_{r} \rho_{i}$. The main drive mechanism is in this case the perpendicular compressibility of the flow.

The low frequency regime $\Omega \leq \Omega_{D} K \rho_{i}$ is more intriguing. Admitting that the source term $\left(S_{2,-1}+S_{2,1}\right)$ is of the order of $S^{\prime}$ times a factor $f_{b a l}$ representative of the level of turbulence ballooning, one gets the estimate

$$
\frac{\phi_{1, \Omega}}{B_{e q}}=\frac{\phi_{-1, \Omega}}{B_{e q}}=-i K_{r}^{2} \rho_{i}^{2} f_{b a l} \frac{\Pi_{R S}(\Omega)}{(1+\tau) \Omega}
$$

No convergence is found for $\Omega \rightarrow 0$ unless the Reynolds stress frequency spectrum vanishes at low frequency, which is unlikely. The same behavior occurs for zonal flows. This indicates a lack of dissipative processes, probably due to a break-down of the theory in absence of particle trapping. We note however that the ratio $\frac{\phi_{1, \Omega}}{\phi_{0, \Omega}}$ is regular and of the order of $K_{r} \rho_{i}$. The poloidal asymmetry is of the form $\cos \theta$, i.e. in-out, and the drive appears to be the asymmetry of the turbulent stress tensor due to turbulence ballooning.

\subsection{Numerical solution}

We assume that the source terms are separable in energy, $\Omega$ and $M$, i.e. we prescribe the following form

$$
\left\{\left[\tilde{\bar{v}}_{E} \cdot \nabla \tilde{\bar{g}}\right]\right\}=F_{e q} s_{M} \mathcal{S}(\tilde{\Omega})
$$

where $s_{-M}=s_{M}$ is real, and the frequency spectrum $\mathcal{S}(\tilde{\Omega})$ is chosen as a Lorentzian

$$
\mathcal{S}(\tilde{\Omega})=\frac{1}{\pi} \frac{\Delta \tilde{\Omega}}{\Omega^{2}+\Delta \tilde{\Omega}^{2}}
$$

All matrix $\mathcal{E}_{M M^{\prime}}$ and source elements $S_{M}$ can then be expressed as combinations of the functions $L_{j}(\Omega)$. The latter can be calculated numerically using a collocation method (see appendix B )[3, 7]. Inverting numerically the matrix $\mathcal{E}$ then provides the values of $h=\mathcal{E}^{-1} \cdot S$. In the following, parameters are safety factor $q=1.5$, normalised radial wavenumber $K_{r} \rho_{i}=0.1$, ion to electron temperature ratio $\tau_{i}=1$, amplitude of source term $s_{0}=-1$, sideband ratios $s_{1} / s_{0}=s_{-1} / s_{0}=0.5$, and frequency width of the source $\Delta \tilde{\Omega}=0.1$.

Fig.1 shows the frequency spectra of $\Omega h_{0, \Omega}$ and $\Omega h_{1, \Omega}$. A GAM signature appears clearly at high frequency, while zonal flows and poloidal convective cells appear at low frequencies. The level of poloidal convective cells is about $10 \%$ lower than zonal flows, in agreement with the estimate above, i.e. $\left|\frac{\Omega h_{1, \Omega}}{\Omega h_{0, \Omega}}\right| \sim$ 

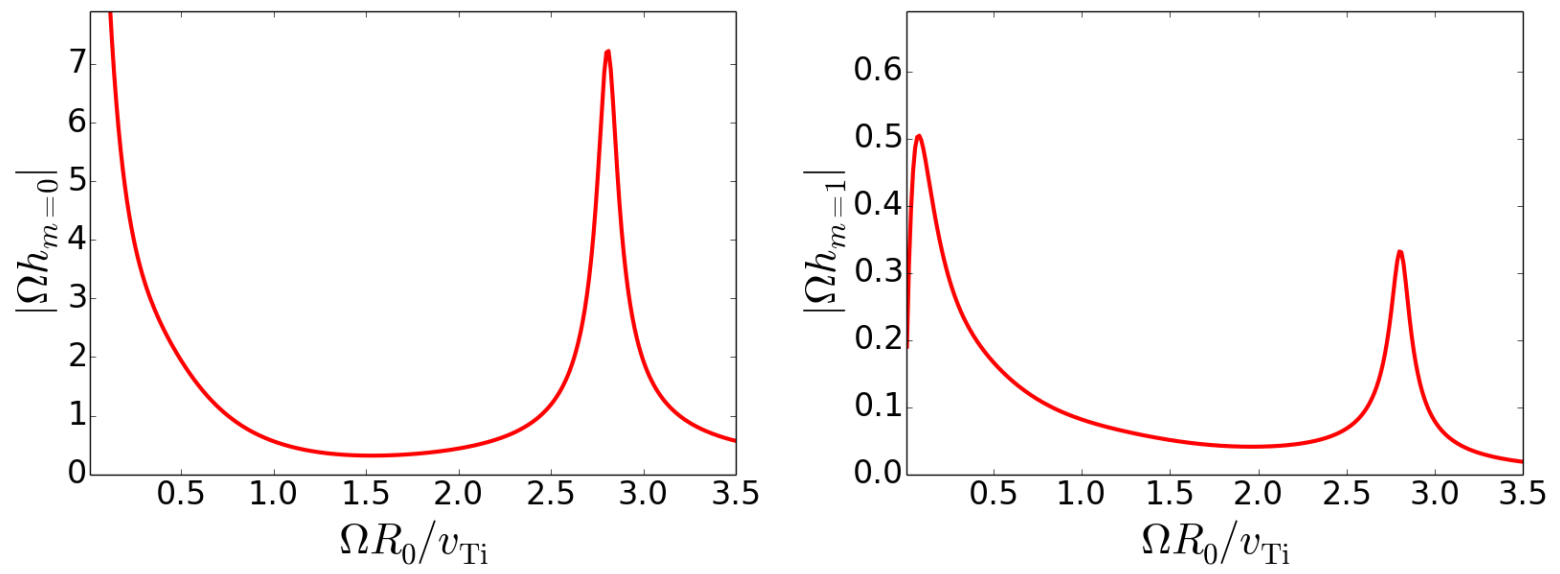

Figure 1: Absolute value of $\Omega h_{0, \Omega}$ and $\Omega h_{1, \Omega}$

$K_{r} \rho_{i}$. Fig.2 and Fig.3 show respectively the ratio $\frac{h_{1, \Omega}}{h_{-1, \Omega}}$ and the phase of $\frac{h_{1, \Omega}}{h_{0, \Omega}}$. It appears that the ratio $\frac{h_{1, \Omega}}{h_{-1, \Omega}}$ is about -1 in the GAM range, while the angle of $\frac{h_{1, \Omega}}{h_{0, \Omega}}$ is $90^{\circ}$ (up-down asymmetry). At lower frequency $\frac{\Omega q R_{0}}{v_{T}} \sim K_{r} \rho_{i}$, the ratio $\frac{h_{1, \Omega}}{h_{-1, \Omega}}$ is again close to -1 , i.e. up-down asymmetry, but with a polarity closer to $-90^{\circ}$, i.e. opposite to GAMs. The very low frequency limit $\frac{\Omega q R_{0}}{v_{T}} \leq\left(K_{r} \rho_{i}\right)^{2}$ is more difficult to assess. The collocation method imposes to choose a frequency mesh with the same mesh size as the velocity variable. Low frequency then means a highly resolved velocity space, and therefore a large computation time. The difficulty is circumvented by increasing the value of $K_{r} \rho_{i}$ up to 0.25 . It can then be checked in Fig. 4 that the ratio $\frac{h_{1, \Omega}}{h_{-1 \Omega}}$ is close to 1 at vanishing frequency, in agreement with a mostly in-out asymmetrical poloidal convective cells. In summary, GAMs and convective cells in the intermediate frequency range are up-down asymmetrical, while low frequency cells are in-out asymmetrical. It also stressed that the polarity of a poloidal convective cell is rarely as clean cut as a GAM, i.e. it varies continuously with the frequency.

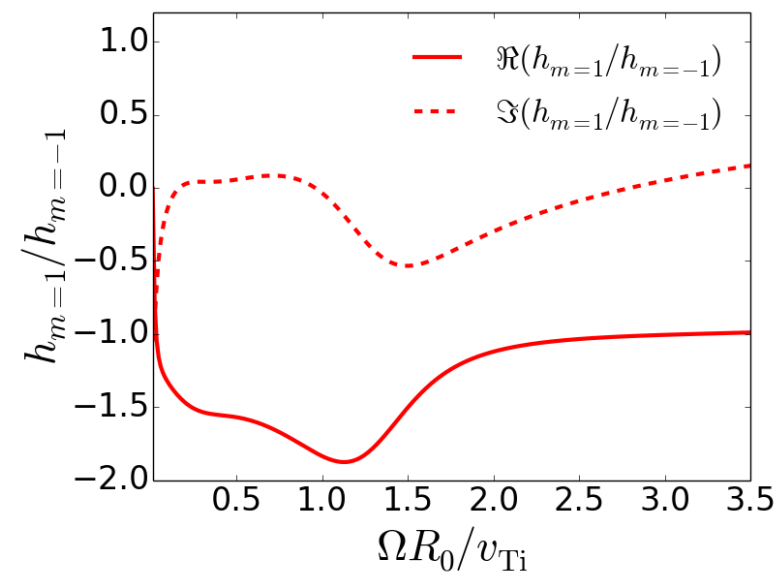

Figure 2: Real and imaginary parts of the ratio $\frac{h_{1, \Omega}}{h_{-1, \Omega}}$ vs $\Omega$

\subsection{Limits of the model}

The model does not account for trapped particles. This is visible by considering the limit $\Omega_{D} \lesssim \Omega_{\text {and }}$ looking at the dynamics of the mode $M=0$. In this case, at leading order in $\tilde{K}=K_{r} \rho_{i}$, one gets

$$
i \Omega h_{0, \Omega} \operatorname{det} \mathcal{E}=X_{0}^{2} S^{\prime}
$$

where

$$
\operatorname{det} \mathcal{E} \simeq\left(\frac{N_{e q}}{T_{e q}}\right)^{3}(1+\tau)^{2} \tilde{K}^{2}\left[1+\frac{q^{2}}{2}\left(1+\frac{\pi}{2(1+\tau)}+i \frac{\sqrt{2 \pi}}{\tilde{\Omega}}\right)\right]
$$




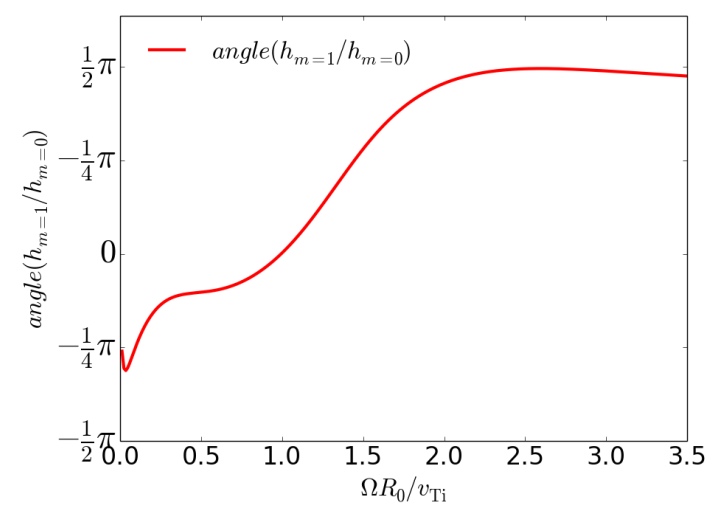

Figure 3: Phase of $\frac{h_{1, \Omega}}{h_{0, \Omega}}$

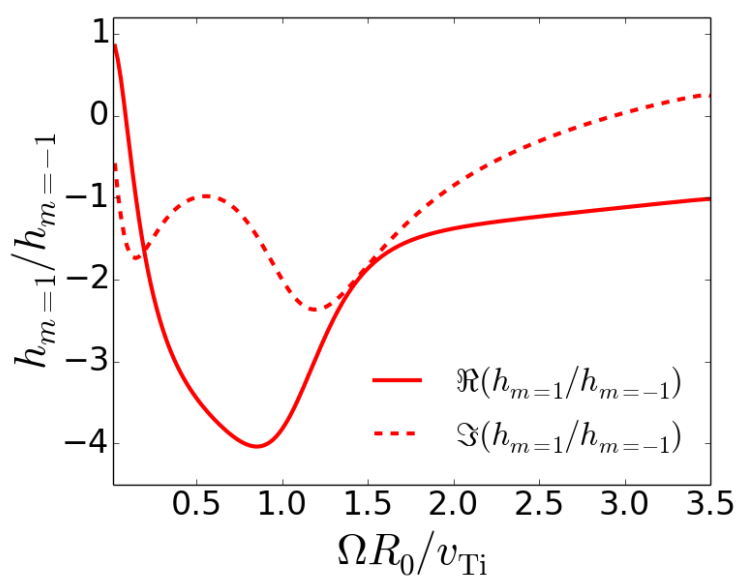

Figure 4: Real and imaginary parts of the ratio $\frac{h_{1, \Omega}}{h_{-1, \Omega}}$ vs $\Omega$ for $K_{r} \rho_{i}=0.25$

Since the source $S^{\prime}$ is equal to $\tilde{K}^{2} \frac{N_{e q} e B_{e q}}{T_{e q}} \Pi_{R S, 0}(\Omega)$, where $\Pi_{R S}(t)$ is the $M=0$, component of the turbulent Reynolds stress at scale $K$, one finds the following equation for the zonal flow

$$
\left[1+\frac{q^{2}}{2}\left(1+\frac{\pi}{2(1+\tau)}\right)\right] \frac{\partial}{\partial t}\left(\frac{\phi_{0}}{B_{e q}}\right)=-\Pi_{R S, 0}(t)-\sqrt{\frac{\pi}{2}} \frac{q v_{T}}{R_{0}} \frac{\phi_{0}}{B_{e q}}
$$

The right hand side of the equation is the expected value with a neoclassical damping in plateau regime. The factor in front of the time derivative corresponds to the renormalisation of inertia due to the passing particles. It differs from the fluid classical value $1+2 q^{2}[15]$ since the prefactor of $q^{2}$ is of the order of 1 , i.e. twice smaller. If the trapped particles were taken into account, this factor should be $1+1.6 \frac{q^{2}}{\sqrt{\epsilon}}[18]$, where $\epsilon=\frac{r}{R_{0}}$.

Trapping is important when the frequency $\Omega$ is of the order of the bounce frequency $\frac{v_{T}}{q R_{0}} \sqrt{\epsilon}$. If we want the present model to be valid for $\Omega \sim \Omega_{D}$, this implies $K_{r} \rho_{i}>\frac{\sqrt{\epsilon}}{q}$. This obviously excludes large scale flows, and requires small values of the inverse aspect ratio. The very low frequency regime $\Omega \sim \Omega_{D} K_{r} \rho_{i}$ seems even more difficult to reach since is requires $K_{r}^{2} \rho_{i}^{2}>\frac{\sqrt{\epsilon}}{q}$, which would only occur for highly corrugated flows in the radial direction. We also recall that the non linear term $\left[\left\langle\overline{\mathbf{v}}_{E}\right\rangle_{\varphi} \cdot \nabla\langle\bar{g}\rangle_{\varphi}\right]_{M \Omega} \simeq 0$, which represents the advection by the convective cell of the associated distribution function, was ignored in this analysis, though it may play some role in the dynamics. Recent numerical simulations actually suggest that its amplitude is comparable to the Reynolds stress tensor $S^{\prime}$ in some cases [2]. 


\section{Conclusion}

a comprehensive calculation of zonal flows, GAMs and poloidal convective cells over a large range of frequencies, for a prescribed turbulent source. Poloidal convective cells are related to poloidal asymmetries of the electric potential at low frequency. They which advect matter via their associated $E \times B$ drift velocity. These asymmetries have to be differentiated from Geodesic Acoustic Modes, which are coherent modes, with a well defined polarity. The calculation is based on a linearised solution of the gyrokinetic Vlasov equation for a prescribed spectrum of helical fluctuations, coupled to a Poisson equation. Two mechanisms have been identified that generate poloidal convective cells. The first one is due to flow compressibility, that results in a pumping of $N=0, M= \pm 1$ modes by $N=0, M=0$ zonal flows - i.e. a sideband effect. In this case, the main drive is the flux surface averaged Reynolds stress, as for zonal flows. It is found to dominate over a large range of frequencies, down to the curvature frequency. A signature of this mechanism is a polarity that is mainly an up-down asymmetry. A second mechanism comes from turbulence ballooning, which induces in turn a ballooning of the Reynolds stress. This mechanism prevails at very low frequency, and produces in-out asymmetrical convective cells. Poloidal convective cells are Landau damped, a property shared with GAMs, but not with zonal flows. The consequence is an amplitude that is lower than zonal flows. The typical ratio of poloidal convective cells to zonal flow amplitude is of order $K_{r} \rho_{i}$. Therefore it is expected that these cells will play an important role in region where zonal flows are intense, for instance staircases [6]. Both analytical and numerical solutions of the coupled Vlasov-Poisson problem substantiate this analysis. It is stressed however that the results found in the very low frequency regime are questionable as trapped ions are not included in this analysis - the complexity of such a calculation is beyond the scope of this paper. Also, non linear self-interaction terms are discarded. These terms do not exist for zonal flows, but are finite for convective cells, and may play a role in their non linear dynamics.

\section{Acknowledgments}

Authors would like to thank the organizers of the "Festival de Théorie" held in July 2017 in Aix-en-Provence, France, who allowed fruitful discussions for this work. This work has been carried out within the framework of the EUROfusion Consortium and has received funding from the Euratom research and training program 2014-2018 under grant agreement No 633053 for the project WP17-ENR-CEA-02. 


\section{A Flux of vorticity}

drive of poloidal convective cells $\mathcal{J}\left[\overline{\mathbf{v}}_{E} \cdot \nabla \bar{g}\right]_{M \Omega}$ is computed. This source term can be recast as

$$
\mathcal{J}\left[\overline{\mathbf{v}}_{E} \cdot \nabla \bar{g}\right]_{M \Omega}=\mathcal{J}\left[\frac{\mathbf{B}_{e q}}{B_{e q}^{2}} \cdot(\nabla(\mathcal{J} \phi) \times \nabla \bar{g})\right]_{M \Omega}
$$

The gradient of any field can be written as $\nabla \phi=\frac{\partial \phi}{\partial \theta} \nabla \theta+\frac{\partial \phi}{\partial \psi} \nabla \psi+\frac{\partial \phi}{\partial \alpha} \nabla \alpha$. Let us note that

$$
\frac{\partial \phi}{\partial \theta}|\nabla \theta| \ll \frac{\partial \phi}{\partial \psi}|\nabla \psi|, \frac{\partial \phi}{\partial \alpha}|\nabla \alpha|
$$

in the frame of the ballooning representation, i.e. the gradient of fluctuations along the field lines is much smaller than the transverse gradients. This also implies that transverse gradients of fluctuations are larger than gradients of metric elements or of any equilibrium quantity. Another consequence is that the gyroaverage operator $\mathcal{J}$ commutes with quantities that depend on $\theta$ only. This implies that $\nabla(\mathcal{J} \phi) \times \nabla \bar{g}$

$$
\nabla(\mathcal{J} \phi) \times \nabla \bar{g}=\left(\frac{\partial(\mathcal{J} \phi)}{\partial \alpha} \frac{\partial \bar{g}}{\partial \psi}-\frac{\partial(\mathcal{J} \phi)}{\partial \psi} \frac{\partial \bar{g}}{\partial \alpha}\right) \mathbf{B}_{e q}
$$

It appears that the gyroaverage operator $\mathcal{J}$ commutes with derivatives with respect to $\Psi\left(\mathcal{J} \partial_{\psi} f=\partial_{\psi} \mathcal{J} f\right)$ and $\alpha\left(\mathcal{J} \partial_{\alpha} f=\partial_{\alpha} \mathcal{J} f\right)$. Then is is possible to show that $\mathcal{J}\left[\overline{\mathbf{v}}_{E} \cdot \nabla \bar{g}\right]_{M \Omega}=\frac{\partial \Gamma_{\Pi}}{\partial \psi}$

$$
\Gamma_{\Pi}=\int \frac{d \theta}{2 \pi} e^{-i M \theta+i \Omega t} \int \frac{d \alpha}{2 \pi} \mathcal{J}\left(\overline{\mathcal{E}}_{\alpha} \bar{g}\right)
$$

where $\overline{\mathcal{E}}_{\alpha}=\frac{\partial(\mathcal{J} \phi)}{\partial \alpha}$. We note that $\bar{g}$ can be replaced safely by $\bar{f}$ since the adiabatic response does not contribute to the flux as $\int \frac{d \alpha}{2 \pi} \overline{\mathcal{E}}_{\alpha}(\mathcal{J} \phi)=0$.

\section{B Treatment of the resonant functions $L_{j}(\Omega)$ and numerical solution}

functions are expanded at lowest order in $K^{2}$. It then appears that the matrix $\mathcal{E}$ and the source $\{S\}$ can be expressed as

$$
\begin{aligned}
& \mathcal{E}_{a}=(1+\tau)\langle 1\rangle_{v}+L_{0}-L_{2} \\
& \mathcal{E}_{b}=K^{2}+2 L_{2} \\
& \mathcal{E}_{c}=L_{1} \\
& \mathcal{E}_{d}=-L_{2}
\end{aligned}
$$

and

$$
\begin{aligned}
-i \Omega\{S\}_{M=-1} & =\left(-i L_{1}+L_{0}-2 L_{2}\right) \mathcal{S}(\Omega) s_{-1} \\
-i \Omega\{S\}_{M=0} & =\left(-1+2 L_{2}\right) \mathcal{S}(\Omega) s_{0} \\
-i \Omega\{S\}_{M=1} & =\left(i L_{1}+L_{0}-2 L_{2}\right) \mathcal{S}(\Omega) s_{1}
\end{aligned}
$$

where

$$
L_{j}(\Omega)=\left\langle\frac{\Omega^{2-j} \Omega_{D}^{j}}{\Omega_{+} \Omega_{-}+2 \Omega_{D}^{2}}\right\rangle
$$

and the prefactor $\frac{N_{e q}}{T_{e q}}$ has been removed in all kinetic integrals. The potential $\{h\}$ is given by the relation $\{h\}=\mathcal{E}^{-1}\{S\}$.

The dimensionless functions $L_{j}(\Omega)$ can be expressed in normalized form as

$$
L_{j}(\tilde{\Omega})=\left(\frac{\tilde{\Omega}}{q}\right)^{2-j} \int_{0}^{+\infty} d u e^{-u} \int_{-\infty}^{+\infty} \frac{d \zeta}{\sqrt{\pi}} e^{-\zeta^{2}} \frac{\bar{\Omega}_{D}^{j}}{R}
$$


where $u=\frac{1}{2} \frac{v_{\perp}}{v_{T}^{2}}, \zeta=\frac{1}{\sqrt{2}} \frac{v_{\|}}{v_{T}}, R=\frac{2}{q^{2}} \zeta^{2}+2 \bar{\Omega}_{D}^{2}-\left(\frac{\tilde{\Omega}}{q}\right)^{2}$, and $\bar{\Omega}_{D}=\frac{\tilde{K}}{2}\left(u+2 \zeta^{2}\right)$. These are resonant integrals, which must be continued analytically to ensure a proper inverse Laplace transport (Landau prescription). This is most easily done by introducing an elliptical change of variables

$$
\begin{aligned}
& \zeta(\rho, \theta)=\frac{q}{\sqrt{2}} \tilde{K} \rho \sin \theta \\
& u(\rho, \theta)=\sqrt{2} \rho \cos \theta-\tilde{K}^{2} q^{2} \rho^{2} \sin ^{2} \theta
\end{aligned}
$$

The quantities of interest (magnetic drift frequency, resonance, and energy) have still simple expressions in this set of variables

$$
\begin{aligned}
& R(\rho, \theta)=\tilde{K}^{2} \rho^{2}-\left(\frac{\tilde{\Omega}}{q}\right)^{2} \\
& \bar{\Omega}_{D}(\rho, \theta)=\frac{\tilde{K}}{\sqrt{2}} \rho \cos \theta \\
& E(\rho, \theta)=u+\zeta^{2}=\sqrt{2} \rho \cos \theta-\frac{1}{2} \tilde{K}^{2} q^{2} \rho^{2} \sin ^{2} \theta
\end{aligned}
$$

The Jacobian of the change of this variables is $J=q \tilde{K} \rho$. The resonant integrals Eq.(18) can then be recast in the following convenient form

$$
L_{j}(\varpi)=\frac{q \tilde{K}}{\sqrt{\pi}} \varpi^{2-j} \int_{0}^{+\infty} d \rho \frac{2 \rho}{\rho^{2}-\varpi^{2}} \int_{0}^{\theta_{0}(\rho)} d \theta e^{-E} \bar{\Omega}_{D}^{j}
$$

where the angle $\theta_{0}(\rho)$ is such that $\cos \theta_{0}=-a+\sqrt{1+a^{2}}, a=\frac{1}{\sqrt{2} \tilde{K}^{2} q^{2} \rho}, \bar{\Omega}_{D}=\frac{1}{\sqrt{2}} \rho \cos \theta$, and $\varpi=\frac{\tilde{\Omega}}{q \tilde{K}}$. The cut-off $\theta \leq \theta_{0}$ ensures that the perpendicular energy $u$ is always positive. For positive and real values of $\tilde{K}$, the Laplace contour prescription in $\Omega$ translates to the same one on the normalised variable $\varpi$. Hence the integrals to be calculated are of the form

$$
L_{j}(\varpi)=\varpi^{2-j} \int_{0}^{+\infty} d \rho \frac{2 \rho G_{j}(\rho)}{\rho^{2}-\varpi^{2}}
$$

where the functions

$$
G_{j}(\rho)=\frac{q \tilde{K}}{\sqrt{\pi}} \int_{0}^{\theta_{0}(\rho)} d \theta e^{-E} \bar{\Omega}_{D}^{j}
$$

are smooth in $\rho$. This formulation allows an easy implementation of the Landau prescription by using the identity

$$
\frac{2 \rho}{\rho^{2}-\varpi^{2}}=\frac{1}{\rho-\varpi}+\frac{1}{\rho+\varpi}
$$

Restricting the analysis to $\Re(\Omega)>0$, one find the rule

$$
L_{j}(\varpi)= \begin{cases}\varpi^{2-j} \int_{0}^{+\infty} d \rho \frac{2 \rho G_{j}(\rho)}{\rho^{2}-\varpi^{2}} & \Im(\varpi)>0 \\ \varpi^{2-j} P . P . \int_{0}^{+\infty} d \rho \frac{2 \rho G_{j}(\rho)}{\rho^{2}-\varpi^{2}}+i \pi \varpi^{2-j} G_{j}(\varpi) & \Im(\varpi)=0 \\ \varpi^{2-j} \int_{0}^{+\infty} d \rho \frac{2 \rho G_{j}(\rho)}{\rho^{2}-\varpi^{2}}+2 i \pi \varpi^{2-j} G_{j}(\varpi) & \Im(\varpi)<0\end{cases}
$$

The principal part that appears in the function $L_{0}$ exhibits a logarithmic singularity near $\varpi=0$ that is regularized by the $\varpi^{2}$ prefactor . The functions $L_{1}$ and $L_{2}$ are regular near $\varpi=0$. The functions $L_{j}$ can be computed in the domain $\Re(\tilde{\Omega})<0$ by using the properrties $L_{j}(-\tilde{\Omega})=\left[L_{j}(\tilde{\Omega})\right]^{*}$ for positive $\tilde{K}$. All these integrals are calculated numerically by using a collocation integration method $[3,7]$. The asymptotic forms Eq.(10) are recovered when $\tilde{\Omega} \geq 1$ with an excellent accuracy.

\section{References}

[1] C Angioni and P Helander. Neoclassical transport of heavy impurities with poloidally asymmetric density distribution in tokamaks. Plasma Physics and Controlled Fusion, 56(12):124001, 2014.

[2] Y. Asahi, V. Grandgirard, Y. Sarazin, P. Donnel, X. Garbet, Y. Idomura, G. Dif-Pradalier, and G. Latu. Synergy of turbulent and neoclassical transport through poloidal convective cells. To be submitted in Plasma Physics and Controlled Fusion, 2018. 
[3] M. Brambilla and R. Bilato. Advances in numerical simulations of ion cyclotron heating of nonmaxwellian plasmas. Nuclear Fusion, 49(8):085004, 2009.

[4] J. W. Connor, J. B. Taylor, and H. R. Wilson. Shear damping of drift waves in toroidal plasmas. Phys. Rev. Lett., 70:1803-1805, Mar 1993.

[5] P H Diamond, S-I Itoh, K Itoh, and T S Hahm. Zonal flows in plasmas - a review. Plasma Physics and Controlled Fusion, 47(5):R35, 2005.

[6] G. Dif-Pradalier, G. Hornung, Ph. Ghendrih, Y. Sarazin, F. Clairet, L. Vermare, P. H. Diamond, J. Abiteboul, T. Cartier-Michaud, C. Ehrlacher, D. Estève, X. Garbet, V. Grandgirard, Ö. D. Gürcan, P. Hennequin, Y. Kosuga, G. Latu, P. Maget, P. Morel, C. Norscini, R. Sabot, and A. Storelli. Finding the elusive $\mathbf{E} \times \mathbf{B}$ staircase in magnetized plasmas. Phys. Rev. Lett., 114:085004, Feb 2015.

[7] R. Dumont. Private communication.

[8] D. Esteve, Y. Sarazin, X. Garbet, V. Grandgirard, S. Breton, P. Donnel, Y. Asahi, C. Bourdelle, G. DifPradalier, C. Ehrlacher, C. Emeriau, Ph. Ghendrih, C. Gillot, G. Latu, and C. Passeron. Self-consistent gyrokinetic modeling of neoclassical and turbulent impurity transport. Nuclear Fusion, 58(3):036013, 2018.

[9] X Garbet, Y Asahi, P Donnel, C Ehrlacher, G Dif-Pradalier, P Ghendrih, V Grandgirard, and Y Sarazin. Impact of poloidal convective cells on momentum flux in tokamaks. New Journal of Physics, 19(1):015011, 2017.

[10] T. S. Hahm, M. A. Beer, Z. Lin, G. W. Hammett, W. W. Lee, and W. M. Tang. Shearing rate of time-dependent exb flow. Physics of Plasmas, 6(3):922-926, 1999.

[11] K. Hallatschek and D. Biskamp. Transport control by coherent zonal flows in the core/edge transitional regime. Phys. Rev. Lett., 86:1223-1226, Feb 2001.

[12] F. L. Hinton and R. D. Hazeltine. Theory of plasma transport in toroidal confinement systems. Rev. Mod. Phys., 48:239-308, Apr 1976.

[13] F L Hinton and M N Rosenbluth. Dynamics of axisymmetric exb and poloidal flows in tokamaks. Plasma Physics and Controlled Fusion, 41(3A):A653, 1999.

[14] F. L. Hinton and Marshall N. Rosenbluth. Transport properties of a toroidal plasma at low-tointermediate collision frequencies. The Physics of Fluids, 16(6):836-854, 1973.

[15] S.P. Hirshman. The ambipolarity paradox in toroidal diffusion, revisited. Nuclear Fusion, 18(7):917, 1978.

[16] M. McIntyre. On global-scale atmospheirc circulations. "Perspective in fluid dynamics" Cambridge university press, 2000.

[17] F. Romanelli and F. Zonca. The radial structure of the ion temperature gradient driven mode. Physics of Fluids B: Plasma Physics, 5(11):4081-4089, 1993.

[18] M. N. Rosenbluth and F. L. Hinton. Poloidal flow driven by ion-temperature-gradient turbulence in tokamaks. Phys. Rev. Lett., 80:724-727, Jan 1998.

[19] T.-H. Sugama, H.and Watanabe. Collisionless damping of geodesic acoustic modes. Journal of Plasma Physics, 72(6):825 to 828, 2006.

[20] Z. X. Wang, J. Q. Li, J. Q. Dong, and Y. Kishimoto. Generic mechanism of microturbulence suppression by vortex flows. Phys. Rev. Lett., 103:015004, Jul 2009.

[21] W.Connor, R.J. Hastie, and J.B. Taylor. High mode number stability of an axisymmetric toroidal plasma. Proceedings of the Royal Society of London A: Mathematical, Physical and Engineering Sciences, 365(1720):1-17, 1979. 
[22] Niels Winsor, John L. Johnson, and John M. Dawson. Geodesic acoustic waves in hydromagnetic systems. The Physics of Fluids, 11(11):2448-2450, 1968.

[23] S. K. Wong and V. S. Chan. Self-consistent poloidal electric field and neoclassical angular momentum flux. Physics of Plasmas, 16(12):122507, 2009.

[24] D. Zarzoso, X. Garbet, Y. Sarazin, R. Dumont, and V. Grandgirard. Fully kinetic description of the linear excitation and nonlinear saturation of fast-ion-driven geodesic acoustic mode instability. Physics of Plasmas, 19(2):022102, 2012.

[25] Fulvio Zonca, Liu Chen, and Robert A Santoro. Kinetic theory of low-frequency alfven modes in tokamaks. Plasma Physics and Controlled Fusion, 38(11):2011, 1996. 